\title{
Konzeption und Anlage der Studie
}

In diesem Kapitel werden die Konzeption und die Anlage der empirischen Studie dargestellt. Dazu erfolgt zunächst eine Konkretisierung der Fragestellung. Darauf aufbauend werden im nachfolgenden Abschnitt die Anlage und der Ablauf der Studie beschrieben, bevor die Erhebungsinstrumente, die eingesetzten Materialien sowie die Methoden zur Datenauswertung eingehend erläutert werden.

\subsection{Konkretisierung der Fragestellung}

Die dargestellten theoretischen Perspektiven zeigen auf, dass die Fähigkeit bestehendes Wissen in neuen Situationen zu aktivieren und anzuwenden, im Wesentlichen von der Qualität der vorhandenen Wissensstrukturen abhängt. Sie müssen tragfähig, hinreichend vernetzt und flexibel sein, um auf neue Anforderungssituationen übertragen werden zu können. Insbesondere im Hinblick auf ein fortgesetztes Mathematiklernen erfordert dies eine stetige Weiterentwicklung, Reorganisation und Anpassung vorhandener mentaler Wissensrepräsentationen. Aus mathematikdidaktischer Perspektive kann der Aufbau tragfähiger mentaler Modelle als Ausbilden von Grundvorstellungen beschrieben werden. In diesem Zusammenhang wurde herausgestellt, dass es hilfreich erscheint, Transfer nicht als ein Produkt von Lernen im Sinne einer abgeschlossenen Transferleistung zu betrachten, sondern als einen Prozess der Übertragung und des Anknüpfens an vorhandene Wissensstrukturen zu konzeptualisieren:

Elektronisches Zusatzmaterial Die elektronische Version dieses Kapitels enthält Zusatzmaterial, das berechtigten Benutzern zur Verfügung steht https://doi.org/10.1007/978-3-658-33981-4_3. 
Ein Transferprozess ist der Prozess der Anwendung oder Übertragung mathematischer Begriffe, Verfahren und Strukturen auf eine neue Anforderungssituation. Diese Prozesse können umfassen:

- Den Transfer zwischen Sach- und Anwendungskontexten,

- den Transfer zwischen verschiedenen Darstellungen und Repräsentationsebenen sowie

- das Herstellen und Begründen von mathematischen Zusammenhängen.

Ausgehend von dieser Definition lassen sich die Forschungsfragen der empirischen Studie auf normativer und deskriptiver Ebene formulieren. Die erste Frage betrifft die normative Beschreibung von Transferprozessen im Rahmen von sachanalytischen Überlegungen:

Frage 1: Welche Transferprozesse sind zur Lösung einer neuen Anforderung aus normativer Sicht erforderlich und welche Grundvorstellungen werden dafür benötigt?

- Worin besteht der konzeptuelle Kern als Gegenstand eines Transfers, d.h. welche Begriffsaspekte, Verfahren oder Strukturen sollen übertragen werden?

- Welche Transferprozesse sind auf Ebene des Sachkontexts respektive der mit ihnen verbundenen situativen Handlungskonzepte sowie zwischen Darstellungen und Repräsentationsebenen erforderlich?

- Welche möglichen Schwierigkeiten, Übergeneralisierungen und fehlerhafte Übertragungen sind zu erwarten?

Die Diskussion verschiedener Theorien und Erklärungsmodelle von Transfer führt zu der Annahme, dass zum Aufbau von tragfähigen Grundvorstellungen zum Bruchzahlbegriff verschiedene Transferprozesse zur Erweiterung und Vernetzung von Begriffsaspekten erforderlich sind. Zudem kann angenommen werden, dass diese Transferprozesse sich sehr individuell, d.h. in Abhängigkeit von individuellen Erklärungsmodellen und über einen längeren Zeitraum entwickeln und sich in den Interaktionen von Lernenden dokumentieren und analysieren lassen.

Hinsichtlich der deskriptiven Untersuchung im Rahmen von interaktiven Bearbeitungsprozessen von Schülerinnen und Schülern im Mathematikunterricht kann das zentrale Forschungsinteresse der Studie in den folgenden Forschungsfragen konkretisiert werden. Diese betreffen die Rekonstruktion von Transferprozessen und der zugrundeliegenden individuellen Deutungs- und Erklärungsmodelle der Lernenden (Frage 2), den Vergleich der auf normativer Ebene intendierten Transferprozesse mit 
den auf deskriptiver Ebene rekonstruierten Transferprozessen der Lernenden (Frage 3) sowie den Zusammenhang zwischen Transferprozessen und der Entwicklung von Grundvorstellungen von Brüchen und dem Umgang mit ihnen (Frage 4).

Frage 2: Welche Transferprozesse können in den individuellen Bearbeitungsprozessen der Lernenden dokumentiert werden?

- Welche transferrelevanten individuellen Erklärungsmodelle und Deutungen von Brüchen und dem Umgang mit Brüchen können in den Bearbeitungsprozessen der Lernenden rekonstruiert werden?

Frage 3: Inwieweit entsprechen die individuellen Transferprozesse der Lernenden den intendierten Transferprozessen?

- Welche Zusammenhänge können zwischen den Transferprozessen und den individuellen Deutungs- und Erklärungsmodellen der Lernenden hergestellt werden?

Frage 4: Worauf sind etwaige Divergenzen zwischen den intendierten Transferprozessen und den in den Bearbeitungen der Lernenden dokumentierten Transferprozessen zurückzuführen?

- Inwieweit kann eine Beziehung zwischen ausbleibenden, negativen und nicht intendierten Transferprozessen und fehlerhaften Deutungs- und Erklärungsmodellen der Lernenden hergestellt werden?

\subsection{Konzeption und Anlage der empirischen Studie}

Vor dem Hintergrund des dargestellten Forschungsinteresses und der herausgearbeiteten Forschungsdesiderata wird die Studie in einem authentischen Unterrichtszusammenhang mit Schülerinnen und Schülern einer fünften Klasse eines städtischen Gymnasiums durchgeführt. Mithilfe von Bild- und Tonaufzeichnungen werden die Partnerarbeiten der Schülerinnen und Schüler erfasst, die zusammen mit den schriftlichen Arbeitsprodukten die Datengrundlage für qualitative Analysen der Bearbeitungsprozesse darstellen. Es wird ein qualitatives Untersuchungsdesign gewählt, da das Kerninteresse der Untersuchung in der detaillierten Analyse der Bearbeitungen und Lösungsprozesse liegt. Durch die Analyse der Prozessdaten der Lernen- 
den sollen Transferprozesse identifiziert und weiterführenden Analysen zugänglich gemacht werden.

Studiendesign: Grundlage für die Studie ist eine Unterrichtseinheit zur Einführung des Bruchzahlbegriffs, die in den regulären Mathematikunterricht einer fünften Klasse eingebunden ist ${ }^{1}$. Die Unterrichtseinheit wird vom regulären Fachlehrer der Klasse durchgeführt und es werden keine außerplanmäßigen oder zusätzlichen Unterrichtsstunden in Anspruch genommen. Zur Kontrolle der im Unterricht eingesetzten Materialien und Aufgaben wurden die Inhalte, Themen und der Verlauf der Unterrichtseinheit mit dem Mathematiklehrer besprochen und in Form eines Arbeitsbuches festgehalten, das das reguläre Lehrwerk für die Unterrichtseinheit ersetzt. Zu Beginn der Unterrichtseinheit wird mit einem Vortest das Vorwissen der Schülerinnen und Schüler zu elementaren Grundkenntnissen und Vorstellungen zu Bruchzahlen erhoben. Zur Dokumentation der individuellen Lernfortschritte wird die Unterrichtseinheit mit einem parallelisierten Nachtest abgeschlossen.

Im Rahmen des Unterrichts werden zu drei Zeitpunkten Datenerhebungen durchgeführt. Die Datenerhebungen sind mit der Einführung bestimmter Inhaltsaspekte koordiniert und finden im Rahmen von Doppelstunden mit einem Zeitumfang von 90 Minuten statt. In diesen Doppelstunden arbeiten die Schülerinnen und Schüler in Paaren, die über alle drei Erhebungszeitpunkte möglichst beibehalten werden. Die Zusammenstellung der Paare wird auf Grundlage der persönlichen Präferenzen der Schülerinnen und Schüler vorgenommen um möglichst harmonische und hemmungsarme Paarkonstellationen zu erhalten. Dadurch sollen soziale Aspekte des Lernprozesses hervorgehoben werden, die eine regelmäßige Interaktion und somit die Gewinnung authentischer Daten gewährleisten sollen.

Die Erhebungssitzungen sind in eine Lern- und eine Anwendungsphase geteilt. In der Lernphase erarbeiten die Schülerinnen und Schüler einen zentralen konzeptionellen Inhalt anhand von interaktiven animierten Lösungsbeispielen, deren Verarbeitung von fokussierenden Fragestellungen unterstützt wird. Der Übergang in die Anwendungsphase wird durch die Bearbeitung von unvollständigen Lösungsbeispielen (,fading examples“) gestaltet, die analog zu den Lösungsbeispielen der Einführung sind und in denen Teile des Lösungsweges ausgeblendet sind, die von den Schülerinnen und Schülern ergänzt werden sollen. In der Anwendungsphase bearbeiten die Schülerinnen und Schüler verschiedene Übungs- und Transferaufgaben, in denen die Inhalte der Lernphase auf neue Anforderungssituationen über-

\footnotetext{
${ }^{1}$ Da das schulinterne Curriculum die Inhalte der durchgeführten Unterrichtseinheit auf die Jahrgangsstufen 5 und 6 aufteilt, wurde in Kooperation mit dem Fachlehrer, der Schulleitung und den Eltern der Schülerinnen und Schülern eine Ausnahmeregelung getroffen.
} 
tragen werden sollen. Die Transferaufgaben gehen dabei über die Reproduktion der Inhalte der Lernphase hinaus und erfordern Transferprozesse auf Ebene des Sachkontexts und der Darstellung.

Untersuchungsgruppe: Die Untersuchungsgruppe stellen 17 Schülerinnen und 13 Schüler einer fünften Klasse eines städtischen Gymnasiums. Abgesehen von der Behandlung von Alltagsbrüchen haben die Schülerinnen und Schüler keinen systematischen Unterricht zum Bruchzahlbegriff erhalten.

Die Schülerinnen und Schüler der Klasse werden durch den Klassenlehrer als eine sehr heterogene Gruppe beschrieben, in der das Leistungsspektrum im Fach Mathematik von sehr starken bis zu sehr schwachen Leistungen abgedeckt wird. Eine Grundvoraussetzung für die Auswahl der Untersuchungsgruppe ist eine hohe Bereitschaft zur Interaktion miteinander sowie gute kommunikative Fähigkeiten.

Entwicklung der Unterrichts- und Studienmaterialien: Die Unterrichts- und Studienmaterialien wurden hinsichtlich eines sukzessiven Aufbaus von Grundvorstellungen zu Bruchzahlen erstellt. Auf Grundlage eines Schulbuchs (vom Hofe, Humpert, Griesel \& Postel, 2012a) wurde ein Arbeitsbuch zusammengestellt, das die Grundlage für den Klassenunterricht stellt. Neben dem Arbeitsbuch und den Studienmaterialien werden keine weiteren Arbeitsmittel eingesetzt. Auf diese Weise wird die Sequenz der Unterrichtsinhalte festgelegt und die Einheitlichkeit der genutzten Repräsentationen und Darstellungen sichergestellt. Die Materialien der Lern- und Anwendungsphasen in den Datenerhebungen sind ebenfalls im Arbeitsbuch enthalten. Die digitalen animierten Lösungsbeispiele für die Lernphasen der Erhebungssitzungen sind als Begleitmaterial für das Schulbuch entwickelt worden, das dem Arbeitsbuch zugrunde liegt. Lediglich die unvollständigen Beispiele sind nicht Inhalt des Arbeitsbuchs, diese wurden auf Grundlage der Lösungsbeispiele konzipiert.

Die Aufgaben im Vor- und Nachtest stammen zu einem großen Teil aus pilotierten Aufgabensammlungen (Pekrun et al., 2006; Pallack, vom Hofe \& Salle, 2013; Salle, 2015) und wurden durch wenige weitere Items ergänzt.

Datenerhebung: Die Vor- und Nachtests werden mit einem Zeitumfang von $45 \mathrm{~min}$ im Klassenunterricht bearbeitet. Die Erhebungssitzungen werden in zwei Computerarbeitsräumen durchgeführt, in denen die Schülerpaare vor jeweils einem Computer arbeiten, an dem die Schülerinnen und Schüler über die gesamte Zeit Zugriff auf das digitale animierte Lösungsbeispiel der Lernphase haben und eigenständig mit diesem arbeiten und im weiteren Sitzungsverlauf auf diese zurückgreifen können. Die Bearbeitung der unvollständigen Beispiele sowie der weiterführenden Trans- 
feraufgaben erfolgt in einem Arbeitsheft in Papierform, sodass die schriftlichen Bearbeitungen der Schülerinnen und Schüler erfasst werden können. Während der gesamten Erhebungssitzung werden durch eine Webcam und Mikrofone die Interaktionen der Partnerarbeiten in Bild und Ton aufgezeichnet, wodurch sie gemeinsam mit den schriftlichen Schülerprodukten die Datengrundlage für qualitative Analysen zur Rekonstruktion der Bearbeitungsprozesse der Schülerinnen und Schüler darstellen. Zur Förderung der Interaktion zwischen den Schülerinnen und Schülern werden sie während der Erhebungssitzungen regelmäßig daran erinnert, dass sie die Aufgaben nicht allein bearbeiten sollen, sondern sich mit ihren Partnerinnen und Partnern über die Lösungswege und Ergebnisse austauschen und diese einander erklären sollen.

In den Phasen der Datenerhebung ist eine zweite Lehrkraft anwesend, sodass in allen Räumen, in denen die Schülerinnen und Schüler arbeiten eine Ansprechperson anwesend ist, die bei technischen Problemen oder organisatorischen Fragen helfen kann und die Arbeitsphase überwacht. Die anwesenden Lehrkräfte geben, anders als im Regelunterricht, keine Hinweise und Tipps für die Lösung von Aufgaben. Sie dürfen einzig darauf hinweisen, dass die Schülerinnen und Schüler sich noch einmal das Lösungsbeispiel anschauen können.

Einbettung in den Klassenunterricht: Durch die Einbindung der Studie in den Klassenunterricht soll die Authentizität der erhobenen Daten sicher gestellt werden. Der Unterricht wird durch den regulären Mathematiklehrer der Klasse durchgeführt. Die Schüler arbeiten, wie sie es gewohnt sind, in unterschiedlichen Arbeitsformaten, d.h. Einzel-, Partner- und Gruppeneinheit sowie im Plenum. In Partner- und Gruppenarbeiten während des regulären Unterrichts wird nicht darauf geachtet, dass die Schülerinnen und Schüler stets in denselben Paarzusammensetzungen arbeiten, dies ist lediglich für die Phasen der Datenerhebungen vorgesehen.

Durch die kooperative Arbeit in den Erhebungssitzungen sollen authentische Interaktionen der Lernenden gefördert werden, die eine Möglichkeit bieten sollen Einsichten in komplexe Unterrichtssituationen und Daten zu individuellen Bearbeitungs- und Argumentationsprozessen zu gewinnen, die deskriptive Analysen individuellen Lernprozesse ermöglichen.

\subsubsection{Anlage der empirischen Studie}

In diesem Abschnitt werden die Durchführung der empirischen Studie und die Datenerhebung beschrieben. Dazu werden zunächst der Ablauf der Studie und die 
inhaltliche Strukturierung der Unterrichtseinheit dargestellt. Es schließen Beschreibungen der Erhebungsinstrumente und Methoden zur Datenerhebung an.

\subsubsection{Ablauf der Studie und inhaltliche Strukturierung der Unterrichtseinheit}

Im Folgenden werden die einzelnen Phasen der Studie in chronologischer Reihenfolge (Abb. 3.1) dargestellt und die konkreten Unterrichtsinhalte näher beschrieben.

1. Erhebung des Vorwissens: Zu Beginn der Unterrichtseinheit wird das Vorwissen der Schülerinnen und Schüler zu Brüchen in einem schriftlichen Test erhoben. Dieser dient einerseits zur Charakterisierung des Leistungsstands der Schülerinnen und Schüler zu Beginn der Unterrichtseinheit. Zum Anderen soll die Erhebung des Vorwissensstandes die Einordnung der in der erhobenen Prozessdaten ermöglichen. Die Konstruktion des Tests wird in Abschnitt 3.3.1 näher erläutert. Den Lernenden stehen 45 Minuten für die Bearbeitung des Vortests zur Verfügung.

2. Arbeit im Klassenunterricht: Im Anschluss an die Erhebung des Vorwissens wird im Klassenunterricht anhand des Arbeitsbuches im Rahmen von zwei Unterrichtsstunden die Herstellung von Stammbrüchen erarbeitet. Die Bildung des Anteils wird an Kreis-, Rechtecks- sowie weiteren zweidimensionalen und dreidimensionalen Repräsentationen veranschaulicht. Es wird zudem das Ergänzen von Stammbrüchen zu einem Ganzen thematisiert.

3. Erste Datenerhebung im Rahmen der Einführung von echten Brüchen: Die Schülerinnen und Schüler erarbeiten in Paaren anhand eines interaktiven animierten Lösungsbeispiels die Herstellung von echten Brüchen. Dabei wird der Begriff des Stammbruchs erweitert und die Bruchherstellung um den zweiten Operator zum Vervielfachen eines Teils ergänzt. Es werden die zuvor verwendeten Repräsentationen aufgegriffen und um die Darstellung der Bruchherstellung Pfeildiagrammen erweitert, die die Herstellungshandlungen als einen aus zwei Teiloperatoren zusammengesetzten Prozess veranschaulichen. In den Übungs- und Transferaufgaben sollen die Schülerinnen und Schüler Brüche und ihre Herstellung ikonisch darstellen, Brüche aus ikonischen Darstellungen ablesen und ihrer Herstellung erläutern, zwischen verschiedenen ikonischen Bruchdarstellungen übersetzen und echte Brüche zu einem Ganzen ergänzen. Die Schülerpaare arbeiten 90 Minuten lang in einem Arbeitsheft und mit einem Computer, auf dem sie jederzeit Zugriff auf das interaktive animierte Lösungsbeispiel haben. 


\begin{tabular}{|c|c|}
\hline Vortest & $45 \mathrm{~min}$ \\
\hline $\begin{array}{l}\text { Klassenunterricht: } \\
\text { Bruch als Teil eines Ganzen - Stammbrüche }\end{array}$ & $90 \mathrm{~min}$ \\
\hline $\begin{array}{l}\text { 1. Datenerhebung: } \\
\text { Bruch als Teil eines Ganzen - Echte Brüche }\end{array}$ & $90 \mathrm{~min}$ \\
\hline $\begin{array}{c}\text { Klassenunterricht: } \\
\text { Bruch als Teil eines Ganzen - Echte Brüche } \\
\text { Bruch als Teil eines Ganzen - Unechte Brüche und } \\
\text { gemischte Schreibweise } \\
\text { Bruch als Teil eines Ganzen - Brüche als Maßzahlen } \\
\text { in Größenangaben } \\
\text { Bruch als Teil mehrerer Ganzer }\end{array}$ & $360 \mathrm{~min}$ \\
\hline $\begin{array}{l}\text { 2. Datenerhebung: } \\
\text { Bruch als Operator - Anteile von beliebigen Größen }\end{array}$ & $90 \mathrm{~min}$ \\
\hline $\begin{array}{l}\text { Klassenunterricht: } \\
\text { Bruch als Operator - Anteile von beliebigen Größen } \\
\text { Äquivalente Bruchzahldarstellungen } \\
\text { Erweitern von Brüchen als Verfeinern einer Einteilung }\end{array}$ & $135 \mathrm{~min}$ \\
\hline $\begin{array}{l}\text { 3. Datenerhebung: } \\
\text { Kürzen von Brüchen als Vergröbern einer Einteilung }\end{array}$ & $90 \mathrm{~min}$ \\
\hline $\begin{array}{l}\text { Klassenunterricht: } \\
\text { Erweitern und Kürzen als Verfeinern und Vergröbern } \\
\text { einer Einteilung } \\
\text { Größenvergleich und Ordnen von Brüchen }\end{array}$ & $180 \mathrm{~min}$ \\
\hline Nachtest & $45 \mathrm{~min}$ \\
\hline
\end{tabular}

Abbildung 3.1 Chronologische Übersicht des Studienablaufs 
4. Arbeit im Klassenunterricht: Anhand des Arbeitsbuchs werden im Rahmen acht Unterrichtsstunden unechte Brüche, Brüche als Maßzahlen in Größenangaben und Brüche als Teile mehrerer Ganzer erarbeitet. Dabei werden unter dem inhaltlichen Aspekt unechte Brüche und gemischte Schreibweise verschiedene Arten der Angabe unechter Brüche erarbeitet. Hierbei wird insbesondere die Verknüpfung der ikonischen und symbolischen Repräsentationsebenen sowie die Umwandlung der unterschiedlichen symbolischen Schreibweisen thematisiert. Zudem wird auch die Ergänzung von unechten Brüchen zum nächsten Ganzen behandelt.

Unter dem Aspekt Brüche als Maßzahlen in Größenangaben werden Brüche als Anteile bekannter Größen eingeführt. Dabei wird das Ganze als Maßeinheit angegeben und Umwandlungen in kleinere Einheiten behandelt. Zur Veranschaulichung werden ikonische Rechtecks- und Streckenrepräsentationen eingesetzt, anhand derer auch Ergänzungen zu einem Ganzen thematisiert werden.

Auf verschiedenen Sachkontexten aufbauend werden im Anschluss anhand von Aufteilungssituationen Brüche als Teile mehrerer Ganzer eingeführt. Die Anteilherstellung wird anhand von ikonischen Repräsentanten konkreter Objekte eingeführt und im weiteren Verlauf auf Maßzahlen erweitert. Zudem wird der Zusammenhang zwischen Brüchen und Divisionsaufgaben hergestellt.

\section{Zweite Datenerhebung im Rahmen der Einführung von Brüchen als Teile} beliebiger Größen: Die Schülerinnen und Schüler erarbeiten in Paaren anhand eines interaktiven animierten Lösungsbeispiels die Bestimmung von Brüchen als Teile beliebiger Größen. Hierbei steht die Grundvorstellung vom Bruch als Operator im Zentrum und das zuvor eingeführte Pfeildiagramm wird aufgegriffen und zur Veranschaulichung und Lösungshilfe zur Bestimmung von Anteilen beliebiger Größen eingesetzt. Es sollen Anteile verschiedener Größen und mehrerer Ganzer bestimmt werden sowie von einem Anteil auf das Ganze geschlossen werden. Auch hier arbeiten die Lernendenpaare 90Minuten lang in einem Arbeitsheft und mit einem Computer, auf dem sie jederzeit Zugriff auf das interaktive animierte Lösungsbeispiel haben.

6. Arbeit im Klassenunterricht: Anhand des Arbeitsbuches wird in drei Unterrichtsstunden das Erweitern von Brüchen als Verfeinern einer Einteilung eingeführt. Dazu werden zunächst äquivalente Brüche thematisiert und anhand ikonischer Bruchdarstellungen erarbeitet, dass Anteile durch beliebige Brüche angegeben werden können. Im Zentrum steht weiterhin das ikonische Verfeinern einer Einteilung, dass abschließend in Beziehung zum symbolischen Erweitern von Brüchen als Multiplikation von Zähler und Nenner mit demselben Faktor gesetzt wird. 


\section{Dritte Datenerhebung im Rahmen der Einführung des Kürzens von Brü- chen als Vergröbern einer Einteilung: In der dritten Doppelstunde zur Datener- hebung erarbeiten die Schülerinnen und Schüler zunächst anhand eines interaktiven animierten Lösungsbeispiels das Verfahren zum Vergröbern einer Einteilung einer ikonischen Bruchdarstellung in einem Rechteck und setzen es mit dem Kürzen von Brüchen auf symbolischer Ebene in Beziehung. Die Einführung des Kürzens bzw. Vergröberns einer Einteilung erfolgt analog zur Einführung des Erweiterns als Verfeinern einer Einteilung. Die nachfolgenden Transferaufgaben thematisieren die Übertragung des Verfahrens auf die ikonische Darstellung in einem Kreis und an einer Strecke, die Übertragung des Erweiterns und Kürzens zur Bestimmung eines Zwischenbruchs im Sachkontext und zum Größenvergleich und Ordnen von Brüchen, das bis zu diesem Zeitpunkt nicht im Unterricht behandelt wurde.}

8. Arbeit im Klassenunterricht: In vier weiteren Unterrichtsstunden im Klassenunterricht vertiefen die Schülerinnen und Schüler anhand des Arbeitsbuches die Verfahren zum Erweitern und Kürzen und erarbeiten verschiedene Strategien zum Größenvergleich von Brüchen als letzten inhaltlichen Teil der Unterrichtseinheit. Dazu stellen sie Brüche als Punkte auf einem Zahlenstrahl dar, vergleichen ikonische Bruchdarstellungen, bestimmen Brüche, die kleiner oder größer als vorgegebene andere Brüche sind, und vergleichen Brüche auf symbolischer Ebene.

9. Nachtest: Für den Nachtest steht den Lernenden eine Unterrichtsstunde mit 45 Minuten zur Verfügung. Neben parallelen Items zum Vortest enthält der Nachtest weitere Items, in denen die Schülerinnen und Schüler bestimmte Sachverhalte begründen sollen.

\subsubsection{Arbeitsbuch für die Unterrichtseinheit}

Die gesamte Unterrichtseinheit wird unter Verwendung eines Arbeitsbuches durchgeführt, das das reguläre Mathematikbuch für diesen Zeitraum ersetzt. Das Arbeitsbuch wurde in Absprache mit der unterrichtenden Lehrkraft entsprechend der vorgesehenen inhaltlichen Strukturierung auf Grundlage der Lehrbücher MATHEMATIK HEUTE 5 (vom Hofe, Humpert, Griesel \& Postel, 2012a) und MATHEMATIK HEUTE 6 (vom Hofe et al., 2012b) zusammengestellt. Das Arbeitsbuch umfasst folgende Inhalte:

1. Teile von Ganzen: Zerlegen eines Ganzen in Halbe, Drittel, Viertel, ... (vom Hofe, Humpert, Griesel \& Postel, 2012a, S. 170 ff.) 
2. Teile von Ganzen: Vielfache von Halben, Dritteln, Vierteln, ... Zähler kleiner als Nenner (vom Hofe, Humpert, Griesel \& Postel, 2012a, S. 173 ff.)

3. Brüche als Maßzahlen in Größenangaben (vom Hofe, Humpert, Griesel \& Postel, 2012a, S. 179f.)

4. Brüche als Teile mehrerer Ganzer (vom Hofe, Humpert, Griesel \& Postel, 2012a, S. $181 \mathrm{f}$.)

5. Bestimmen eines Teils einer beliebigen Größe (vom Hofe, Humpert, Griesel \& Postel, 2012a, S. 183 ff.)

6. Derselbe Anteil - verschiedene Brüche: Gleichheit von Anteilen - Brüche mit gleichem Wert (vom Hofe, Humpert, Griesel \& Postel, 2012a, S. 186)

7. Erweitern eines Bruchs (vom Hofe, Humpert, Griesel \& Postel, 2012a, S. 187 f.)

8. Kürzen eines Bruchs (vom Hofe, Humpert, Griesel \& Postel, 2012a, S. $189 \mathrm{f}$.)

9. Darstellen von Bruchzahlen am Zahlenstrahl (vom Hofe, Humpert, Griesel \& Postel 2012b, S. 63 f.)

10. Vergleichen und Ordnen von gewöhnlichen Brüchen (vom Hofe, Humpert, Griesel \& Postel 2012b, S. 65 ff.)

11. Addieren und Subtrahieren von Brüchen mit gleichem Nenner (vom Hofe, Humpert, Griesel \& Postel, 2012a, S. $191 \mathrm{f}$.)

12. Vermischte und komplexe Übungen (vom Hofe, Humpert, Griesel \& Postel, 2012a, S. 193)

13. Was du gelernt hast (mit Ergänzungen zu vom Hofe, Humpert, Griesel \& Postel, 2012a, S. 194)

14. Bist du fit? (vom Hofe, Humpert, Griesel \& Postel, 2012a, S. 195)

Das Arbeitsbuch dient dem Lehrer als Grundlage für den Unterricht und enthält alle in der Studie verwendeten Lösungsbeispiele als statische Abbildungen sowie alle in den Arbeitsheften zur Datenerhebung verwendeten Aufgabenstellungen. In Ergänzung zu dem verwendeten Arbeitsbuch stehen der Lehrkraft zusätzlich die interaktiven animierten Lösungsbeispiele zu den im Arbeitsbuch enthaltenen statischen Lösungsbeispielen zu Präsentationszwecken zur Verfügung. In der Unterrichtseinheit werden keine zusätzlichen (Arbeits-) Materialien eingesetzt.

\subsection{Erhebungsinstrumente}

In diesem Abschnitt werden die Instrumente dargestellt, mit denen in der Studie Daten erhoben werden. Diese umfassen den Vor- und Nachtest zur Ermittlung des Vorwissens und der Leistungsentwicklung der Lernenden, die Arbeitshefte, in denen 
die Lernenden in den Sitzungen der Datenerhebung arbeiten, sowie das technische Vorgehen zur Videographie der Partnerarbeiten.

\subsubsection{Vor- und Nachtest}

Um zum einen die Vorkenntnisse der Lernenden zu Brüchen im Vorfeld der Unterricht zu beschreiben und den Kenntnisstand der Lernenden zum Abschluss der Unterrichtseinheit zu charakterisieren, wird die Unterrichtseinheit von zwei schriftlichen Tests gerahmt. Zum anderen sollen die Daten der schriftlichen Tests die Einordnung der erhobenen Prozessdaten unterstützen.

Die Konstruktion der Tests erfolgte auf Grundlage von erprobten Testitems aus der Längsschnittstudie PALMA (u. a. Pekrun et al., 2006; Wartha, 2007) und der Studie von Salle (2015). Zur Identifikation weiterer inhaltlicher Kompetenzen wurden unter Berücksichtigung fachdidaktischer Ergebnisse zusätzliche Testitems konzipiert, die jedoch nicht für den Vergleich der Leistungen in Vor- und Nachtest herangezogen werden.

Zum Herstellen der Vergleichbarkeit von Vor- und Nachtest wurden zu den zentralen inhaltlichen Kompetenzen parallele Items bzw. Itemgruppen eingesetzt, deren Lösungshäufigkeiten und Itemschwierigkeiten annähernd gleich sind. Die parallelen Items unterscheiden sich lediglich in den verwendeten Daten und Zahlen. Die parallelisierten Itemgruppen beziehen sich auf die folgenden inhaltlichen Bereiche:

- Ablesen von Bruchteilen echter Brüche aus unterschiedlichen ikonischen Repräsentationen (6 Items)

- Einzeichnen und Einfärben von Bruchteilen in unterschiedlichen ikonischen Repräsentationen (6 Items)

- Ein- und mehrschrittige Sachaufgaben mit Kontexten aus den Bereichen Geldwerte und Längen (3 Items)

- Begründungsaufgaben zum Größenvergleich von Stammbrüchen, echten Brüchen mit gleichem Zähler und anderen Brüchen (3 Items)

- Ergänzen von Multiplikations- und Divisionsoperatoren in Pfeildarstellungen (2 Items)

Neben den parallelisierten Items und Itemgruppen sind im Vortest zusätzliche Items zum Rechnen (4 Items) mit und Ordnen (2 Items) von natürlichen Zahlen enthalten. Der Nachtest enthält zudem zusätzliche Items zu inhaltlichen Bereichen der Unterrichtseinheit. Diese umfassen 
- das Ablesen und unechten Brüchen aus ikonischen Repräsentationen und die Notation in gemischter Schreibweise (3 Items),

- das Einteilen und Einfärben von Anteilen in unterschiedlichen ikonischen Repräsentationen (4 Items),

- das Bestimmen eines Teils mehrerer Ganzer (1 Item),

- Aufgaben zum Erweitern und Kürzen von Brüchen (je 2 Items),

- Begründungsaufgaben zur Äquivalenz von Brüchen (1 Item), zum Größenvergleich von Stammbrüchen (1 Item) und zum Bestimmen einer Zwischenzahl (1 Item).

\subsubsection{Arbeitshefte}

In den Sitzungen der Datenerhebung arbeiten die Schülerinnen und Schüler in Arbeitsheften $^{2}$, die am Ende der Sitzung digitalisiert und in der Folgestunde zurückgegeben werden. Die schriftlichen Bearbeitungen ermöglichen es zusätzliche Erkenntnisse und Einsichten in die konkreten Lernprozesse der Schülerinnen und Schüler zu gewinnen und ergänzen die Videoaufzeichnungen der Kommunikation in den Paararbeiten.

Die Arbeitshefte sind zu allen drei Erhebungssitzungen gleich aufgebaut. Die inhaltliche Sequenz besteht aus einem oder mehreren statischen Lösungsbeispielen mit je einer fokussierenden Fragestellung, zwei unvollständigen Lösungsbeispielen und mehreren Transfer- und Übungsaufgaben (Abb. 3.2).
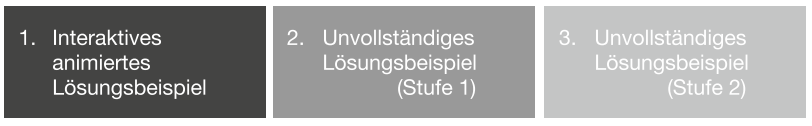
4. Mehrere Transfer- und Übungsaufgaben

Abbildung 3.2 Aufbau einer Sequenz des Arbeitsheftes

Auf den ersten Seiten des Arbeitsheftes sind die Lösungsbeispiele statisch abgebildet, die die Schülerinnen und Schüler in interaktiver und animierter Form am Bildschirm erarbeiten. Die Lösungsbeispiele bilden begriffliche und operative Kerninhalte für die Unterrichtssitzung ab. Ein zentraler Aspekt dieser Lösungsbeispiele ist die Darstellung und Verknüpfung verschiedener Repräsentationen. In Ergänzung zu den digitalen Lösungsbeispielen finden sich in den Arbeitsheften zudem fokus-

${ }^{2}$ Die Arbeitshefte für alle Sitzungen zur Datenerhebung sind im Anhang abgebildet. 
sierende Fragestellungen zur Unterstützung der Verarbeitung der Lösungsbeispiele mitsamt einer Fläche, in der eine Antwort notiert werden soll.

Auf die statische Abbildung des Lösungsbeispiels mit fokussierenden Fragestellungen folgen zwei unvollständige Lösungsbeispiele, in denen die Schülerinnen und Schüler die fehlenden Lösungsschritte ergänzen sollen. Die unvollständigen Lösungsbeispiele sind analog zum Vorgehen im Lösungsbeispiel nach dem Prinzip der "'fading examples“ (siehe Abschnitt 1.2.2 und 3.4.2) gestaltet, sodass im zweiten unvollständigen Beispiel mehr Lösungsschritte ergänzt werden müssen als im ersten.

Das Lösungsbeispiel und die unvollständigen Beispiele stellen die inhaltliche Grundlage für die nachfolgenden Aufgaben zum Üben und Vertiefen (Transferaufgaben).

In den Sitzungen zur Datenerhebung erfolgen alle Aufgabenbearbeitungen, Notizen und ähnliches im Arbeitsheft, sodass dieses alle Aufzeichnungen der Doppelstunde vollständig enthält und somit als Referenz für die Rekonstruktion der Bearbeitungsprozesse herangezogen werden kann.

\subsubsection{Videographie}

Für die Studie sind die konkreten Lern- und Bearbeitungsprozesse der Lernenden von primärem Interesse. Zum Zweck einer möglichst umfassenden Erhebung werden mit der Software MORAE@ Videoaufzeichnungen angefertigt. Die Computerarbeitsplätze der Schüler(-innen)-Paare sind mit Webcams und Mikrofonen ausgestattet. Mit der Software MORAE@ werden simultan die Bildschirme und Bildschirmaktivitäten sowie das Bild der Webcams und der Ton der Mikrofone aufgezeichnet. Die gesammelten Video- und Audiodaten werden synchronisiert in einer Videodatei zusammengeführt.

\subsection{Darstellung der Materialien}

In diesem Abschnitt werden die in der Studie genutzten Materialien erläutert und exemplarisch dargestellt. Die eingesetzten Tests und Arbeitshefte sind im Anhang abgebildet. 


\subsubsection{Interaktive animierte Lösungsbeispiele}

Die interaktiven animierten Lösungsbeispiele basieren auf statischen Lösungsbeispielen, die in den Mathematik-Lehrbüchern MATHEMATIK HEUTE 5 (vom Hofe, Humpert, Griesel \& Postel, 2012a) und MATHEMATIK HEUTE 6 (vom Hofe, Humpert, Griesel \& Postel 2012b) enthalten sind. Die Arbeit mit interaktiven animierten Lösungsbeispielen entspricht nicht dem gewöhnlichen Unterrichtsgeschehen. Dennoch sind (statische) Lösungsbeispiele ein wesentlicher Inhalt der meisten aktuellen Lehrwerke und werden in vielen Fällen dafür eingesetzt, ,,anhand einer prototypischen Aufgabenlösung ein Konzept bzw. einen Begriff auf operative Weise einzuführen oder Lösungsprozeduren oder Prinzipien zu illustrieren“ (Salle, 2015, S. 30). Die Arbeit von Rezat (2009) zeigt, dass Schülerinnen und Schüler Lösungsbeispiele häufig und besonders vielfältig nutzen, z. B. als Hilfe zum Bearbeiten von Aufgaben sowie zum Festigen, Vertiefen und Aneignen von Wissen (Rezat, 2009, S. 252). Die vielfältige Nutzung von Lösungsbeispielen, insbesondere im Vergleich zu anderen Elementen von Schulbüchern, kann dahingehend interpretiert werden, dass die Arbeit mit Lösungsbeispielen bei Schülerinnen und Schülern sehr beliebt ist (vgl. Salle, 2015, S. 32). Für den Einsatz von Lösungsbeispielen in dieser Studie kann angenommen werden, dass die Schülerinnen und Schüler der Untersuchungsgruppe zumindest in Ansätzen mit Lösungsbeispielen und der selbstständigen Arbeit mit ihnen vertraut sind.

Im Hinblick auf die vorliegende Studie werden die interaktiven animierten Lösungsbeispiele vor allem mit dem Ziel der Anregung des kommunikativen Austauschs in den Lernendenpaaren eingesetzt. Die Arbeit von Salle (2015) dokumentiert sehr umfassend, dass der Einsatz digitaler animierter Lösungsbeispiele in Partnerarbeiten die Lernenden zu reichhaltigen Argumentationsprozessen anregen kann. Von den Kommunikationsprozessen, die auf diese Weise angeregt werden, ist zu erwarten, dass sie authentischer sind als Kommunikationsprozesse, die in Interviewsituationen oder mit der Methode des „Lauten Denkens“ (vgl. Chi, Bassok, Lewis, Reimann \& Glaser, 1989) gewonnen werden können. Vor diesem Hintergrund werden sie in dieser Untersuchung vor allem zur Einleitung der Lernphase und zur Initiierung eines authentischen interaktiven Austauschs in den Lernendenpaaren eingesetzt.

Die Grundlagen der Konzeption, Konstruktion und Gestaltung der verwendeten digitalen animierten Lösungsbeispiele werden von Salle (2015) sehr detailliert beschrieben und sollen an dieser Stelle nicht weiter vertieft werden.

Im Folgenden ist der Endzustand eines digitalen animierten Lösungsbeispiels abgebildet (Abb. 3.3). Über eine Navigationsleiste werden die einzelnen Animationen des Lösungsbeispiels gesteuert. 


\section{Mathematik heute ab i Schroedel}

a) Erkläre, wie $\frac{5}{8}$ eines Ganzen entsteht. Stelle das Ganze mit Kreisflächen dar.

Lösung:

Die Kreisfläche (die Pizza) wird in 8 gleich große Teile zerlegt.

Ein Stück davon ist $\frac{1}{8}$.

Davon werden dann 5 Teile genommen. Das sind dann $\frac{5}{8}$.

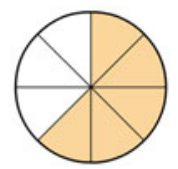

Diesen Ablauf kann man auch mit Pfeilen darstellen:

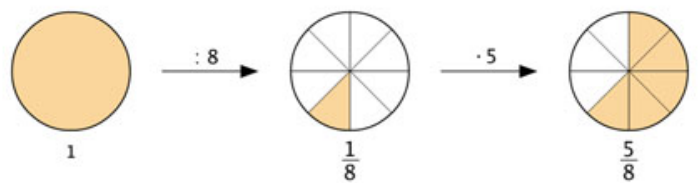

\section{메 (II)}

Abbildung 3.3 Endzustand des animierten Lösungsbeispiels 1a zur anschaulichen Herstellung des Bruchs $\frac{5}{8}$

Die animierten Lösungsbeispiele werden im Aufgabenheft der Lernenden mit jeweils einer fokussierenden Fragestellung begleitet, die sich auf das Kernkonzept des Lösungsbeispiels bezieht. Die fokussierenden Fragestellungen haben einen offenen Charakter um möglichst große Freiheiten für die Beantwortung einzuräumen und sollen ebenfalls dazu dienen, die Kommunikation in den Partnerarbeiten anzuregen.

Die fokussierende Frage zu diesem Lösungsbeispiel zielt zum Beispiel auf das Auslösen einer Diskussion der Bedeutung von Zähler und Nenner einer Bruchzahl ab. Hierbei soll erörtert werden, dass der Nenner eines Bruchs den Grad der Einteilung des Ganzen und der Zähler die Anzahl der markierten Teile repräsentiert. Die fokussierende Frage zum zweiten Teil des Lösungsbeispiels, in dem die anschauliche Herstellung des Bruchs $\frac{3}{8}$ animiert wird, zielt auf eine eigene Formulierung bzw. Beschreibung des gesamten Herstellungsprozesses durch die Lernenden ab. Die Beantwortung der fokussierenden Fragestellungen erfolgt schriftlich im Arbeitsheft.

Prompt zu Lösungsbeispiel 1a: Warum erhält man $\frac{5}{8}$ und nicht $\frac{8}{5}$ ? Begründe. 
Prompt zu Lösungsbeispiel 1b: Beschreibe mit eigenen Worten, wie man aus einem Ganzen $\frac{3}{8}$ erhält.

\subsubsection{Unvollständige Lösungsbeispiele}

Die unvollständigen Beispiele wurden analog zu den jeweiligen Lösungsbeispielen des entsprechenden Inhalts nach dem Prinzip der „fading examples“ (siehe Abschnitt 1.2.2) gestaltet. Das bedeutet, dass die unvollständigen Beispiele genau wie das Lösungsbeispiel aufgebaut sind, jedoch einzelne Lösungsschritte ausgeblendet wurden, sodass sie von den Lernenden ergänzt werden müssen. Im Vergleich zu den korrespondierenden Lösungsbeispielen werden geringe Änderungen bezüglich des Kontexts und der ikonischen Repräsentation vorgenommen. Die Änderungen beschränken sich in den meisten Fällen jedoch auf Zahlenwerte und die verwendeten ikonischen Repräsentationen, sodass der Transfer zwischen Lösungsbeispiel und unvollständigen Beispielen als (sehr) naher Transfer beschrieben werden kann. Im Folgenden werden die auf das zuvor dargestellte interaktive animierte Lösungsbeispiel folgenden unvollständigen Beispiele exemplarisch beschrieben (Abb. 3.4).

Im ersten unvollständigen Beispiel (Aufgabe 1) sollen die Lernenden analog zu den Brüchen $\frac{5}{8}$ (Kreis) und $\frac{3}{8}$ (Rechteck) im Lösungsbeispiel erklären, wie die Brüche $\frac{6}{8}$ (Kreis) und $\frac{4}{5}$ (Rechteck) entstehen. Dazu sind die ikonischen Repräsentationen der einzelnen Herstellungsschritte bereits vorgegeben, d. h. die Lernenden sollen lediglich zu den ikonischen Repräsentationen gehörenden symbolischen Rechenoperatoren und Brüche ergänzen. Im Falle von Unklarheiten der Lernenden in Hinsicht auf die Aufgabe, enthält eine auf eine auszufüllende Lücke weisende Sprechblase den Hinweis „Trage in die Kästen ein, was man rechnen muss, um zum nächsten Schritt zu kommen“.

Das zweite unvollständige Beispiel (Aufgabe 2) ist analog zum Lösungsbeispiel und dem ersten unvollständigen Beispiel gestaltet (Abb.3.5). Anders als im ersten unvollständigen Beispiel sind hier jedoch keine Lösungsschritte mehr vorgegeben, sodass die Lernenden alle Schritte der Bruchherstellung eigenständig darstellen müssen. Zur Strukturierung der Aufgabenlösung wurde hierfür die Aufgabenstellung angepasst, sodass die Lernenden in je zwei Teilaufgaben dazu aufgefordert werden, zu erklären die $\frac{2}{3}$ bzw. $\frac{3}{8}$ eines Ganzen entsteht, wozu sie ein Pfeilbild zeichnen können, und den gesuchten Bruchteil in der vorgegebenen ikonischen Repräsentation einzuzeichnen. Durch den Hinweis in der Aufgabenstellung „Du kannst auch ein Pfeilbild zeichnen“ wird auf das Vorgehen im vorhergehenden Lösungsbeispiel verwiesen, das auf diese Aufgabe übertragen werden soll. 
Eine Pizza wird in 4 gleich große Stücke aufgeschnitten. Sarah nimmt sich 3 solcher Stücke. Das sind 3 Viertel der ganzen Pizza. Statt 3 Viertel schreibt man auch $\frac{3}{4}$.

a) Erkläre, wie $\frac{6}{8}$ eines Ganzen entsteht. Stelle das Ganze durch eine Kreisfläche dar.

\section{Lösung:}

Die Kreisfläche (z.B. ein Kuchen) wird in 8 gleich große Teile zerlegt, davon werden dann 6 genommen.
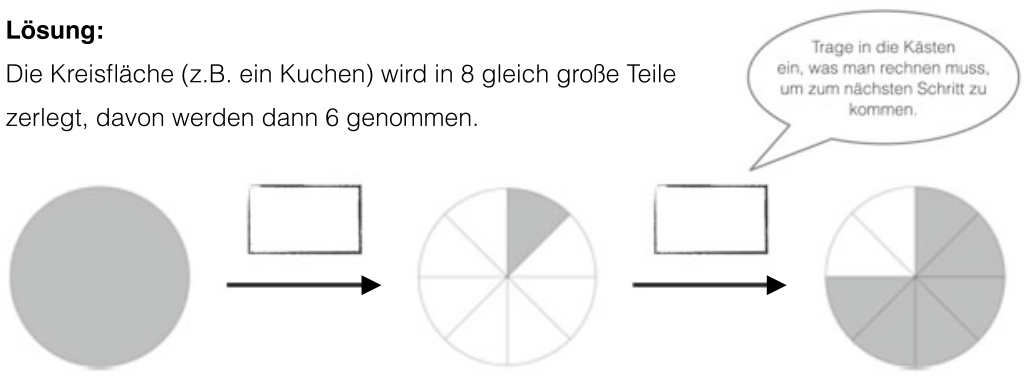

Als Pfeilbild mit Zahlen geschrieben:
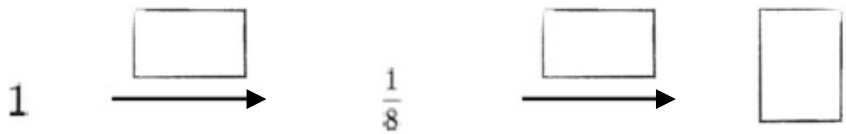

b) Erkläre, wie $\frac{4}{5}$ eines Ganzen entsteht. Stelle das Ganze durch ein Rechteck dar.

\section{Lösung:}

Das Rechteck (z.B. ein Blatt Papier) wird in 5 gleich große Teile zerlegt, davon werden dann 4 genommen.
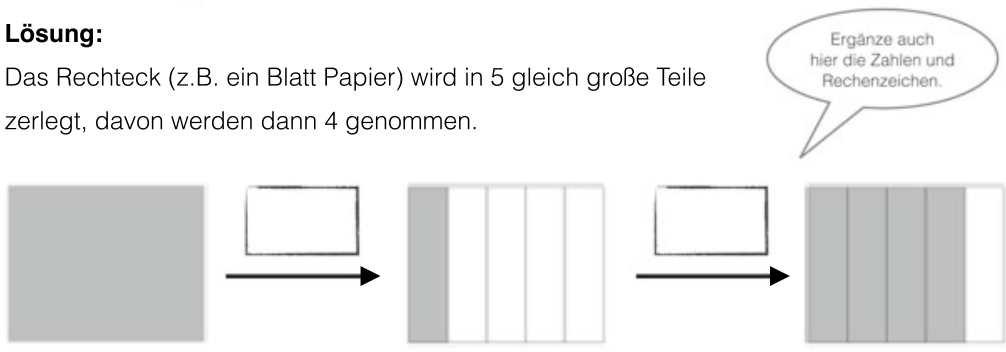

Als Pfeilbild mit Zahlen geschrieben:
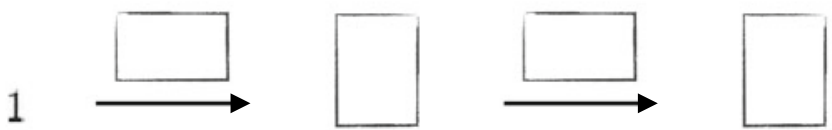

Abbildung 3.4 Erstes unvollständiges Beispiel zur Herstellung der Brüche $\frac{6}{8}$ und $\frac{4}{5}$ 
Ein Kuchen wird in 12 gleich große Stücke aufgeschnitten. Nils nimmt sich 3 solcher Stücke. Das sind 3 Zwölftel des ganzen Kuchens, statt 3 Zwölftel schreibt man auch $\frac{3}{12}$.

a) Das Ganze ist hier durch einen Kreis dargestellt.

1) Erkläre, wie $\frac{2}{3}$ eines Ganzen entsteht. Du kannst auch ein Pfeilbild zeichnen.

2) Zeichne den Bruchteil ein.

\section{Lösung:}

1)

2)
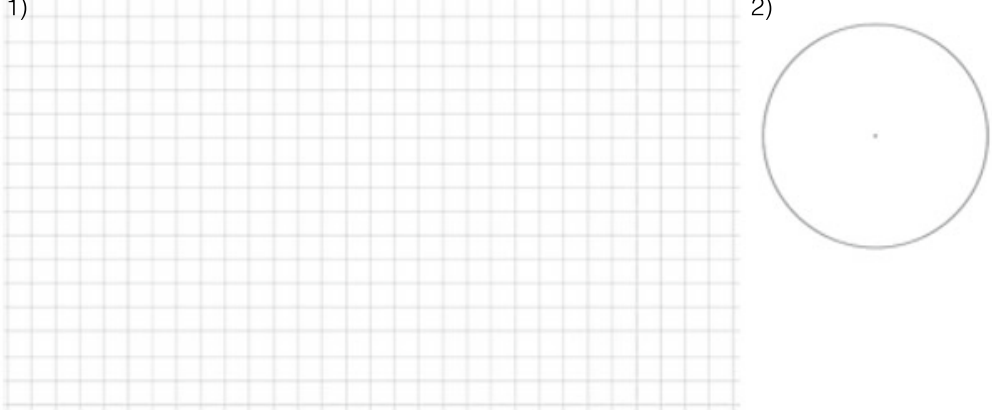

b) Jetzt ist das Ganze ein Rechteck.

1) Erkläre, wie $\frac{3}{8}$ des Ganzen entsteht. Du kannst auch ein Pfeilbild zeichnen.

2) Zeichne den Bruchteil ein.

\section{Lösung:}

1)

2)
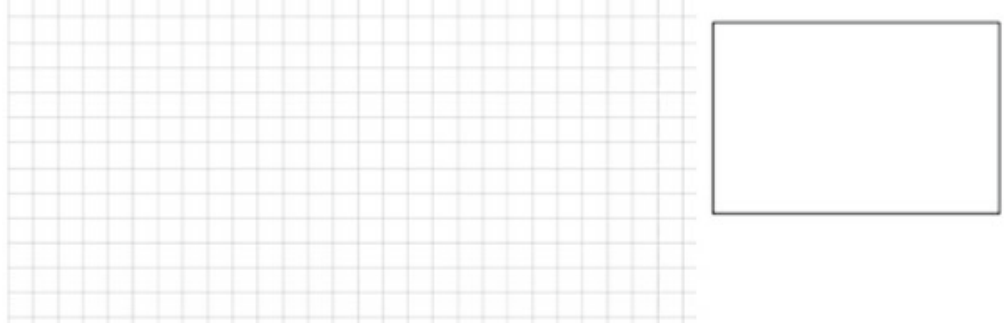

Abbildung 3.5 Zweites unvollständiges Beispiel zur Herstellung der Brüche $\frac{2}{3}$ und $\frac{3}{8}$ 
Im Hinblick auf die Ausbildung von Grundvorstellungen in den unvollständigen Beispielen stehen drei Transferprozesse im Vordergrund:

1. Die Übertragung des Bruchherstellungsverfahrens von den Lösungsbeispielen in eine eigenständige Durchführung (Anwendung des Verfahrens),

2. die Übertragung des Bruchherstellungsverfahrens auf neue Brüche und ikonische Figuren sowie

3. die Übersetzung der Herstellungshandlung in die ikonische und symbolische Repräsentationsebene.

Die zentrale Grundvorstellung in diesen Aufgaben ist die Grundvorstellung Bruch als Operator. Mit der Ausbildung der Operatorvorstellung sind folgende Annahmen verbunden, die in die Konzeption Materialien einbezogen wurden:

- Die Einübung des Bruchherstellungsverfahrens : $n \cdot m$ als mentales Muster führt zum Aufbau einer Grundvorstellung.

- Dieses mentale Muster kann durch eine Generalisierung unabhängig von Zahlen und Repräsentationen angewendet werden.

- Die Darstellung als Pfeilschema in ikonischer und symbolischer Form dient als Hilfe zum Anknüpfen an das Vorwissen der Lernenden.

\subsubsection{Transferaufgaben}

Auf die unvollständigen Beispiele folgen mehrere Transfer- und Übungsaufgaben, die die Kernkompetenzen zu den jeweils eingeführten Begriffsaspekten und Verfahren abdecken. Die Aufgaben unterscheiden sich zum Teil deutlich von den Lösungsbeispielen und unvollständigen Beispielen. Neben dem Arbeitsheft zur Datenerhebung sind die Aufgaben auch im Arbeitsbuch der Schülerinnen und Schüler abgebildet und stammen allesamt aus vom Hofe et al. (2012a; 2012b). Die Aufgaben werden direkt im Arbeitsheft bearbeitet.

Im Folgenden werden zur Veranschaulichung die auf das oben geschilderte Lösungsbeispiel und die unvollständigen Beispiele anknüpfenden Aufgaben kurz erläutert. Eine ausführliche Darstellung der für die Analyse ausgewählten Aufgaben erfolgt an den entsprechenden Stellen der deskriptiven Analysen der Bearbeitungsprozesse im Ergebnisteil.

Aufgaben 1 und 2 - Unvollständige Beispiele: Wie im vorhergehenden Abschnitt geschildert, soll mit den unvollständigen Beispielen in Aufgaben 1 und 2 die 
eigenständige Anwendung des Verfahrens zur Herstellung von Brüchen unterstützt werden. Die Lernenden sollen angeleitet erklären, wie bestimmte Brüche hergestellt werden. Dabei wird besonders die Verknüpfung der Handlung auf enaktivikonischer Ebene mit den entsprechenden Rechenoperationen auf symbolischer Ebene akzentuiert. Die zentralen Transferprozesse sind hier, wie im vorhergehenden Abschnitt geschildert.

Aufgabe 3 - Darstellen von Brüchen: In Aufgabe 3 sollen die Lernenden die Brüche $\frac{3}{4}, \frac{2}{3}, \frac{5}{6}$ und $\frac{3}{10}$ ikonisch in einem Kreis oder Rechteck darstellen. Die didaktische Intention dieser Aufgabe ist vor allem die Übung der Bruchdarstellung. Die Lernenden können sich eigenständig für die Darstellung in einem Kreis oder einem Rechteck entscheiden. Die darzustellenden Brüche wurden so gewählt, dass einerseits weitgehend vertraute Darstellungen der Brüche $\frac{3}{4}$ und $\frac{2}{3}$ angefertigt werden sollen, aber auch Bruchdarstellungen von weniger vertrauten Brüchen, $\frac{5}{6}$ und $\frac{3}{10}$, angefertigt werden sollen. Die zentralen Transferprozesse in dieser Aufgabe sind:

- Die Anwendung des Bruchherstellungsverfahrens zur Anfertigung ikonischer Bruchdarstellungen in einer Kreis- oder Rechtecksrepräsentation und

- Die Übertragung des Verfahrens zur Bruchdarstellung auf weniger vertraute und womöglich bisher unbekannte Brüche.

Aufgabe 4 - Darstellung von Brüchen in einer Strecke: Während die ikonische Darstellung von Brüchen bis zu diesem Zeitpunkt ausschließlich anhand von Kreisund Rechtecksrepräsentationen vorgenommen wurde, sollen die Lernenden in dieser Transferaufgabe ihr Vorgehen auf die lineare Darstellung einer Strecke übertragen. Für jede Teilaufgabe ist eine Strecke mit der Länge von zwölf Kästchen abgebildet, an denen die Lernenden die Brüche $\frac{1}{4}, \frac{2}{3}, \frac{5}{6}$ und $\frac{5}{12}$ darstellen sollen. Es ist somit ein Transfer auf Repräsentationsebene erforderlich, der in zwei Transferschritten beschrieben werden kann:

- Die Übertragung des Teiloperators : $n$ zur Einteilung der Strecke in $n$ gleich große Teile bzw. Abschnitte und

- die Übertragung des Teiloperators $\cdot m$ zum Färben von $m$ Streckenabschnitten.

Aufgabe 5 - Ablesen von Brüchen aus ikonischen Rechtecksdarstellungen und Erläuterung des jeweiligen Herstellungsverfahrens: In Aufgabe 5 sind vier Brüche in Rechtecksrepräsentationen dargestellt. Die Lernenden sollen dazu jeweils angeben, welche Brüche dargestellt sind und erklären, welches Vorgehen den Darstellungen zugrunde liegt. Dazu wird der Hinweis gegeben, dass sie Herstellungs- 
handlung auch in einem Pfeildiagramm erläutern können. Die didaktische Intention dieser Aufgabe ist die Verknüpfung der Anteil- und der Operatorvorstellung. Die Lernenden sollen die abgelesenen Brüche mit ihrer Herstellungshandlung verbinden. Diese Aufgabe dient vornehmlich Übungszwecken. Wohingegen die Lernenden in den vorhergehenden Aufgaben vor allem selber ikonische Darstellungen von Brüchen anfertigen sollten, sollen sie hier Brüche aus Darstellungen ablesen und ihre Herstellung erklären.

\section{Aufgabe 6 - Ablesen von Brüchen aus dreidimensionalen Quaderrepräsenta- tionen: Auch in Aufgabe 6 sollen die Lernenden Brüche aus ikonischen Repräsen- tationen ablesen. Anders als zuvor beinhaltet diese Aufgabe jedoch einen Transfer auf Repräsentationsebene, indem die Lernenden die Brüche nicht aus zweidimen- sionalen Flächenrepräsentationen, sondern aus dreidimensionalen Quaderrepräsen- tationen ablesen sollten. Diese Darstellungsform ist den Lernenden nicht gänzlich unvertraut, da im Arbeitsbuch im Abschnitt zu Stammbrüchen eine ähnliche Auf- gabe enthalten ist, in der die Lernenden Stammbrüche aus dreidimensionalen Qua- derdarstellungen ablesen sollten.}

Aufgabe 7 - Ergänzen eines Bruchteils zu einem Ganzen: In der abschließenden Aufgabe dieses Arbeitsheftes sollen die Lernenden die in symbolischer Form vorgegebenen Brüche $\frac{1}{4}, \frac{5}{8}, \frac{7}{12}$ und $\frac{7}{25}$ zu einem Ganzen ergänzen. Die Aufgabenstellung lautet: „Wie viel fehlt zu einem Ganzen? Du kannst auch zeichnen“.

Die Bestimmung des zu einem Ganzen fehlenden Anteils erfordert im Wesentlichen drei Transferprozesse:

- Bei Stammbrüchen: Die Umdeutung / Interpretation von einem Ganzen als eine aus $n$ Teilen zusammengesetzte Einheit, wobei das n-fache Vielfache einer dieser Teile $\frac{1}{n}$ das Ganze ergibt (vgl. equipartitioning bei Steffe \& Olive, 2010).

- Bei echten Brüchen: Die Anwendung des Bruchherstellungsverfahrens zur Interpretation von echten Brüchen $\frac{m}{n}$ als $m$ Teile eines in $n$ gleiche Teile geteilten Ganzen.

- Die Verknüpfung einer Teil-Ganzen- mit einer Teil-Teil-Ganzen-Relation: Es bedarf einer Verknüpfung zwischen Stammbrüchen und der Einheit 1 um dann eine Verknüpfung zwischen der Anzahl der Teile von echten Brüchen und der Anzahl der Teile des Ganzen herzustellen.

Diese Transferschritte sind im Lernmaterial nicht direkt abgebildet und die Lernenden sind gefordert diese Schritte selbstständig herzuleiten. Zentral ist hierfür die 
individuelle Vorstellungsgrundlage im Bezug auf die Grundvorstellungen Bruch als Anteil und Bruch als Operator.

\subsection{Auswertungsmethoden}

Vor dem Hintergrund des Forschungsinteresses werden quantitative Daten über das Vorwissen und die Leistungsentwicklung der Schülerinnen und Schüler sowie qualitative Daten über die Bearbeitungsprozesse der Schülerinnen und Schüler in Partnerarbeiten erhoben. Im Folgenden werden die Methoden zur Auswertung dieser Daten erläutert.

\subsubsection{Vor- und Nachtest}

Mithilfe des Vor- und Nachtests sollen Informationen über den Kenntnisstand der Schülerinnen und Schüler gewonnen werden. Auf diese Weise lassen sich die Lernentwicklungsverläufe der gesamten Lerngruppe, einzelner Schüler(-innen)-Paare sowie einzelner Schülerinnen und Schüler nachverfolgen und vor dem Hintergrund der Bearbeitungsprozesse einordnen.

Den Kern der Auswertungen bildet eine dichotomisierte Auswertung der parallelen Items, über die ein Vergleich der individuellen Kenntnisstände der Schülerinnen und Schüler zu Beginn und zum Ende der Unterrichtseinheit hergestellt wird. Für diese Auswertungen werden ausschließlich die parallelen Items herangezogen. Dies sind:

- Ablesen von Bruchteilen echter Brüche aus unterschiedlichen ikonischen Repräsentationen (6 Items)

- Einzeichnen und Einfärben von Bruchteilen in unterschiedlichen ikonischen Repräsentationen (6 Items)

- Ein- und mehrschrittige Sachaufgaben mit Kontexten aus den Bereichen Geldwerte und Längen (3 Items)

- Begründungsaufgaben zum Größenvergleich von Stammbrüchen, echten Brüchen mit gleichem Zähler und anderen Brüchen (3 Items)

- Ergänzen von Multiplikations- und Divisionsoperatoren in Pfeildarstellungen (2 Items)

Die prozentuale Testleistung der Lernenden wird über die Anzahl der richtig beantworteten Items und der Gesamtzahl der betreffenden Items errechnet. Durch Sub- 
traktion des Vortestergebnisses vom Ergebnis des Nachtests ergibt die die Differenz der beiden Prozentwerte, die die Veränderung über den Verlauf der Unterrichtseinheit beschreibt.

In Ergänzung zum Vergleich der parallelisierten Testitems werden die Lösungen ausgewählter Sach- und Begründungsaufgaben sowie nicht-paralleler Testaufgaben detaillierter analysiert. Hierdurch sollen einerseits zusätzliche Informationen über das Vorwissen der Schülerinnen und Schüler zu Beginn der Unterrichtseinheit gewonnen werden und andererseits mögliche Auswirkungen der Unterrichtseinheit anhand spezifischer Begründungsaufgaben untersucht werden.

Die nicht-parallelisierten Aufgaben im Vortest umfassen den Umgang und das Rechnen mit natürlichen Zahlen:

- Ordnen von zwei- bis fünfstelligen natürlichen Zahlen (2 Items)

- Komplexe Sachaufgaben zur Addition und Multiplikation natürlicher Zahlen (2 Items)

- Sachaufgabe zum Aufteilen eines Geldbetrags (1 Item)

Die nicht-parallelisierten Items im Posttest betreffen spezifische Kompetenzen zu Bruchzahlen, die Inhalt der Unterrichtseinheit sind.

- Sachaufgabe zur Dichte von Bruchzahlen (1 Item)

- Begründungsaufgaben zur Äquivalenz und zum Größenvergleich von Bruchzahlen (2 Items)

\subsubsection{Analyse von Bearbeitungsprozessen}

Vor dem Hintergrund des Forschungsinteresses dieser Arbeit wird für die Analyse der individuellen Bearbeitungsprozesse der Schülerinnen und Schüler ein qualitativer Zugang gewählt. Das primäre Forschungsinteresse der Datenanalyse ist zu untersuchen, inwieweit sich die normativ intendierten Transferprozesse in den individuellen Lernprozessen der Schülerinnen und Schüler abbilden und worauf etwaige Divergenzen zwischen normativer und deskriptiver Ebene zurückzuführen sind. Somit ist es das Ziel, das Denken der Schülerinnen und Schüler im Rahmen von Transferprozessen zu erfassen und zu untersuchen, inwieweit sich in den individuellen Denkprozessen allgemeine Phänomene und Probleme widerspiegeln, die vor dem Hintergrund der Befunde der psychologischen Transferforschung zu interpretieren sind. Das bedeutet, dass es für die Untersuchung von besonderer Bedeutung ist, die individuellen Strategien, Denkmuster und Vorstellungen bzw. die subjekti- 
ven Wirklichkeiten der Schülerinnen und Schüler möglichst eingehend zu erfassen und zu rekonstruieren. In diesem Zusammenhang argumentiert vom Hofe (1998, S. 259), dass sich ein qualitativer bzw. rekonstruktiv-interpretativer Zugang besonders anbietet,

„wenn man sich aus deskriptiver Sicht dafür interessiert, ob Erklärungsmodelle, mit denen man Lern- bzw. Problemlöseprozesse beschreibt, tatsächlich in den Denkprozessen der Schüler die Rolle spielen, die man theoretisch vermutet.“ (vom Hofe, 1998, S. 259)

Da die Gedanken, mentalen Aktivitäten, Modelle und Vorstellungen von Lernenden nicht direkt beobachtbar sind, ,müssen [sie] aus Indikatoren in einem interpretativen Prozess erschlossen und rekonstruiert werden“ (Bikner-Ahsbahs, 2001, S. 182). Zudem entwickelt sich Wissen (als subjektive Erfahrungsbereiche)

„nicht einfach durch „Verfeinerung und Spezialisierung“ im Gebrauch. Ihre Grenzen [die Grenzen der SEBe] überschreitet das Individuum aktiv entwerfend, erprobend und aushandelnd in Situationen sozialer Interaktion.“ (Bauersfeld, 1983, S. 31, Anmerkung des Autors)

Entsprechend geben die individuellen Bedeutungsaushandlungen in auf Sinnkonstruktion ausgelegten interaktiven Argumentationsprozessen Hinweise auf die gedanklichen Konstruktionen der Lernenden und

„demzufolge müssen Vorstellungen zum Bruchbegriff über die Nachkonstruktion dieses Sinns aus der Perspektive der Interagierenden rekonstruiert werden können.“ (Bikner-Ahsbahs, 2001, S. 182, Hervorhebung des Autors)

Vor diesem Hintergrund erfolgt die Analyse der Prozessdaten der Bearbeitungen der Schülerinnen und Schüler nach einem Verfahren, das Elemente der interpretativen Unterrichtsforschung und der Analyse von Grundvorstellungen auf normativer und deskriptiver Ebene verbindet. In diesem Abschnitt werden die methodologischen Grundlagen der interpretativen Unterrichtsforschung und der Analyse von Grundvorstellungen kurz erläutert und hinsichtlich des Vorgehens in dieser Arbeit konkretisiert.

\section{Grundzüge der interpretativen Unterrichtsforschung}

Die interpretative Unterrichtsforschung ist ein Ansatz der Sozialforschung. Der Begriff „Interpretative Unterrichtsforschung“ bezieht sich als ,Sammelbegriff [...] auf den Untersuchungsgegenstand und den methodischen Ansatz von Forschung“ 
(Schütte, 2009, S.78; vgl. auch Krummheuer \& Naujok, 1999, S. 16). Das Forschungsinteresse liegt in der Erfassung der im Mathematikunterricht ständig neu ausgehandelten Deutungen und Bedeutungszuschreibungen in der Interaktion der Lernenden und Lehrenden. Es wird davon ausgegangen, dass Bedeutungen von mathematischen Begriffen und Verfahren als ,situationsüberdauernde Bedeutungszuschreibungen“" (Schütte, 2009, S. 79) in Interaktionen konstruiert werden, die konstitutiv für ihr Handeln sind. Mit den Methoden der interpretativen Forschung sollen bestimmte Situationen im Unterricht und ihre Merkmale aufgedeckt, beschrieben und auf sie hingewiesen werden. Auf diese Weise sollen spezifische Deutungsund Handlungsstrukturen der Lernenden (und Lehrenden) identifiziert und beurteilt werden (vgl. Krummheuer \& Naujok, 1999). In diesem Zusammenhang übernimmt die interpretative Forschung eine beschreibende Funktion: Es werden theoretische Konstrukte erarbeitet, die helfen sollen alltägliche Handlungsprozesse begründet $\mathrm{zu}$ verstehen. Im Zentrum steht somit das Beschreiben und Verstehen von Lernprozessen. Die hierdurch gewonnen theoretischen Einsichten geben Hinweise zur Identifizierung von Bedingungen für die Verbesserung von Unterricht.

Krummheuer und Naujok (1999) fassen drei Merkmale der Interpretativen Unterrichtsforschung zusammen:

1. „die Fokussierung auf alltägliche Unterrichtsprozesse;

2. das rekonstruktive Vorgehen und

3. die theoretische Grundannahme, daß Lernen, Lehren und Interagieren konstruktive Aktivitäten sind.“ (Krummheuer \& Naujok, 1999, S. 15)

Fokussierung auf alltägliche Unterrichtsprozesse: Der Untersuchungsgegenstand in der interpretativen Unterrichtsforschung ist der alltägliche Mathematikunterricht, der als permanentes interpretatives Geschehen verstanden wird, und durch die wechselseitigen und aufeinander bezogenen Handlungen der Interaktionspartner sukzessive entwickelt wird (vgl. Jungwirth \& Krummheuer, 2008, S. 148). Die Frage ist dabei, wie die an der Interaktion Beteiligten ihren unterrichtlichen Alltag herstellen, um diesen zu verstehen. Im Zentrum stehen dabei die „Regelmäßigkeiten im Umgang mit den fachlichen Inhalten unter dem Aspekt ihrer gemeinschaftlichen Produktion und Produziertheit" (Jungwirth \& Krummheuer, 2008, S. 147).

Die Untersuchung in dieser Arbeit verfolgt das Ziel alltägliche Lernprozesse im Mathematikunterricht zu erheben: Die Lernenden arbeiten in Paaren, in denen sie auch im alltäglichen Unterricht zusammenarbeiten, die Lernenden sind es gewohnt sich neue Inhalte in Partner- und Gruppenarbeit zu erarbeiten und zuletzt entstammen die Materialien einem regulären Schulbuch, das sie für die mehrwöchige Zeit der untersuchten Unterrichtseinheit nutzen. Ein wesentlicher Faktor zu Bewahrung 
der Authentizität und Alltäglichkeit der Bearbeitungsprozesse der Lernenden ist, dass die Lehrperson während der Datenerhebung lediglich eine organisatorische und ordnende Funktion einnimmt und keinerlei inhaltliche Auskünfte, Hinweise oder Bemerkungen gibt.

Rekonstruktives Vorgehen: Ein rekonstruktives Vorgehen bedeutet im Sinne von Bohnsack (2007), dass versucht wird die von Individuen konstruierte Wirklichkeit zu rekonstruieren. Diese Rekonstruktion erfolgt auf Grundlage von Aufzeichnungen von Unterricht, die dazu dienen verbale und nonverbale Handlungen festzuhalten. Diese können Ton- und Videoaufzeichnungen sowie die Arbeitsmaterialien der Lernenden umfassen. Hierbei ist zu bedenken, dass die Ergebnisse von Rekonstruktionen Konstruktionen der Konstruktionen sind, die die handelnden Individuen selbst herstellen:

„Die Besonderheit [...] besteht also darin, [...] dass bereits der Gegenstand dieses [rekonstruktiven] Denkens, eben das soziale Handeln, das Alltagshandeln auf unterschiedlichen Ebenen durch sinnhafte Konstruktionen, durch Typenbildung und Methoden vorstrukturiert ist.“ (Bohnsack, 2007, S.27, Hervorhebung im Original, Anmerkung des Autors)

Die interpretativen Analysen der Bearbeitungs- und Lernprozesse stellen somit Rekonstruktionen von dem dar, was die Lehrenden und Lernenden in den entsprechenden Bedeutungsaushandlungen als (Situations-) Definitionen vorgenommen und damit als Wirklichkeit konstruiert haben.

Der Fokus der vorliegenden Arbeit liegt auf der Rekonstruktion der Bedeutungsaushandlungen der Lernenden. Im Speziellen soll rekonstruiert und beschrieben werden, wie sich Transferprozesse in Lernsituationen darstellen und was die Lernenden in den entsprechenden Situationen übertragen. Ziel ist es diese Transferprozesse aufzudecken, zu identifizieren und zu beschreiben, um sie schließlich zu verstehen.

Produkte von interpretativen Analysen: In der interpretativen Unterrichtsforschung geht es nicht darum eine allgemeingültige, umfassende und alles erklärende Theorie zu entwickeln (vgl. Krummheuer \& Naujok, 1999, S. 105; Naujok, 2000, S.32; Jungwirth \& Krummheuer, 2008, S. 167). Vielmehr soll eine auf den Kontext bezogene Theorie für einen begrenzten Gegenstandsbereich entwickelt werden. Naujok bezeichnet dieses Vorgehen daher als ,lokale Theoriegenese“ und die entstehenden theoretischen Produkte ,eher als Versuche [...] die empirischen Phänomene und Zusammenhänge zwischen diesen zu erklären“ (Naujok, 2000, S. 32). Im Vor- 
dergrund stehen dabei die theoretische Ausdifferenzierung und die Typisierung (vgl. Krummheuer \& Naujok, 1999, S. 24).

Die entwickelten lokalen Theorien sollen die ihnen zugrunde liegenden Daten in angemessener Weise widerspiegeln. Das bedeutet, dass die entwickelten Theorien möglicherweise in anderen Zusammenhängen und Kontexten sinnvoll sein können, z. B. beim Erarbeiten anderer mathematischer Inhalte, in unterschiedlichen Unterrichtskontexten oder in den Lernprozessen von jüngeren oder älteren Lernenden.

Die interpretative Methode - Interaktionsanalyse: Das Verfahren der Interaktionsanalyse besteht aus mehreren Arbeitsschritten, die jedoch nicht als lineare und unveränderliche Abfolge von Interpretationsschritten zu betrachten ist. Das Verfahren steckt vielmehr einen Rahmen ab für einen dynamischen Interpretationsprozess, bei dem es möglich ist, einzelne Interpretationsschritte oder ganze Abfolgen von Interpretationsschritten zu wiederholen (Krummheuer \& Naujok, 1999, S. 68; Krummheuer \& Brandt, 2001, S. 90; Fetzer, 2007, S. 27 ff.; Schütte, 2009, S. 104 ff.).

Nach Voigt (1984) und Bauersfeld et al. (1986) beinhaltet eine Interaktionsanalyse fünf Analyseschritte (vgl. auch Krummheuer \& Brandt, 2001, S. 90; Krummheuer \& Fetzer, 2005, S. 25):

1. Gliederung,

2. Allgemeine Beschreibung nach dem ersten Eindruck,

3. Erzeugung alternativer Interpretationen zu den Einzeläußerungen,

4. Turn-by-Turn-Analyse und

5. Zusammenfassung der Interpretation

Die Gliederung einer Interaktionseinheit dient primär der Übersicht und Strukturierung. Sie kann nach verschiedenen Kriterien vorgenommen werden.

Im Anschluss an die Gliederung erfolgt eine allgemeine Beschreibung nach dem ersten Eindruck, die als ,erste mehr oder weniger spontane und oberflächliche Schilderung" (Krummheuer \& Fetzer, 2005, S. 26) zu verstehen ist. Auf diese Weise soll ein Überblick über die Interaktionseinheit gewonnen und festgehalten werden, wie man ihn spontan interpretiert. Im Rahmen einer Erstzuschreibung wird somit der vermutete ,immanente Sinngehalt“ (Bohnsack, 2010, S. 64) benannt.

Den Kern der Interaktionsanalyse bilden die Schritte der Erzeugung alternativer Interpretationen zu den Einzeläußerungen und die Turn-by-Turn-Analyse. In der Erzeugung alternativer Interpretationen von den Einzeläußerungen werden die Äußerungen und Handlungen der Interaktionspartizipienten sequentiell in der Reihe ihres Vorkommens analysiert und die Konstruktionen der Beteiligten rekonstruiert. Die Interpretation einer Äußerung folgt dabei stets der Frage, auf welche 
Weise die Akteure diese Äußerung interpretieren könnten (vgl. Krummheuer, 2012, S. 237) und es wird versucht ,möglichst viele Verstehensmöglichkeiten zu entwerfen“ (Krummheuer \& Fetzer, 2005, S. 26). Dabei dürfen etwaige Plausibilisierungen nur rückwärtsgewandt erfolgen, damit man sich stets ausschließlich auf diejenigen Informationen bezieht, die auch dem Interaktionspartner zu diesem Zeitpunkt zur Verfügung standen. Die erzeugten Interpretationen müssen sich im weiteren Verlauf des Interpretationsprozesses bewähren (vgl. Krummheuer \& Fetzer, 2005, S. 26). Um Stützungsmöglichkeiten zu erhalten schlagen Krummheuer und Naujok (1999, S. 69f.) vor, potentielle Folgehandlungen für die entwickelten Deutungen zu entwerfen. Sofern eine solche Folgehandlung eintritt, kann diese als eine Stützung der Interpretation verstanden werden und man spricht davon, dass sich die jeweilige Interpretation bewährt.

Zur Einschränkung der entwickelten Deutungsalternativen wird eine Turn-byTurn-Analyse durchgeführt. Dabei geht es darum, zu rekonstruieren, inwieweit der zweite Interaktionspartner die Äußerung des ersten Interaktionspartners gemäß einer der zur ersten Äußerung entwickelten Deutungsalternative interpretiert haben könnte. In seiner Reaktion signalisiert der zweite Interaktionspartner dem ersten, wie er dessen Äußerung gedeutet hat, was wiederum dem ersten Interaktionspartner die Möglichkeit gibt eine sogenannte Reparatur vorzunehmen und die Interpretation seines Interaktionspartners zu korrigieren. Bleibt eine solche Reparatur aus, kann sowohl der erste Interaktionspartner sowie auch der analysierende Wissenschaftler davon ausgehen, dass die beiden Interaktionsteilnehmer sich auf eine geltende Deutung verständigt haben (vgl. Krummheuer \& Fetzer, 2005, S. 28; Tiedemann, 2012, S.93):

„Indem man die Beziehung zwischen den Redezügen zu rekonstruieren versucht, zeichnet man die gemeinsame, Zug um Zug hergestellte Themenentwicklung in der Interaktion nach.“ (Tiedemann, 2012, S.93).

Auf diese Weise können einzelne Deutungsalternativen verworfen und der Umfang der Ausarbeitung reduziert werden. Es kann jedoch auch notwendig sein, dass für die vorausgehende Äußerung eine neue Interpretation entwickelt werden muss.

In der Zusammenfassung der Interpretation werden die tragfähigsten Interpretationen zusammengefasst. Da die ausführliche Darstellung einer vollständigen Interaktionsanalyse sehr umfangreich und für den Leser aufgrund der Vielfalt an Deutungen schwer lesbar ist, wird in Publikationen häufig nur die zusammenfassende Interpretation dargestellt.

Die Zusammenfassungen der Interpretationen werden auch als „Deutungshypothesen“ bezeichnet, ,die sich zum Beispiel [...] auf den Verstehensprozeß einer 
Schülerin im Laufe einer bestimmten Unterrichtsreihe oder auf die Einführung von Begriffen im gesamten beobachteten Mathematikunterricht" (Beck \& Jungwirth, 1999, S. 232) beziehen. In der vorliegenden Arbeit beziehen sich diese Deutungshypothesen explizit auf die Transferprozesse in den Bearbeitungen der Schülerinnen und Schüler im Zusammenhang mit der individuellen Entwicklung von Vorstellungen zu Bruchzahlen.

In dieser Arbeit werden die Deutungshypothesen der analysierten Bearbeitungsprozesse einer Unterrichtseinheit miteinander in Beziehung gesetzt und vergleichend diskutiert, um charakteristische Merkmale und Unterschiede in den Transferprozessen der Lernenden herauszustellen. Die vergleichenden Diskussion sowie bereits die Auswahl der dargestellten Bearbeitungsprozesse folgen dabei dem methodischen Prinzip der Komparation.

Das methodische Prinzip der Komparation: Die komparative Analyse ist als Methode der Vergleichsgruppenbildung ein zentrales Element der Grounded Theory (,,constant comparative method,, Strauss \& Corbin, 1990; vgl. auch Bohnsack, 2010; Kelle \& Kluge, 2010). Sie besteht in einem permanenten Vergleich in allen Stadien und auf allen Ebene der Analyse (Bohnsack, 2010, S. 199; Tiedemann, 2012, S. 75), wodurch sie den gesamten Forschungsprozess bestimmt. Das Ziel der komparativen Analyse ist es zunächst das Spezifische an einem Ausschnitt der Realität erfassen und $\mathrm{zu}$ beschreiben, um danach die Spezifität von ausgewählten Fällen in Beziehung zueinander zu untersuchen (Krummheuer \& Brandt, 2001, S. 82). Auf diese Weise soll der Geltungsbereich der Ergebnisse der Analysen über den Rahmen eines einzelnen Falls erweitert werden, da über den Vergleich von Einzelfällen untereinander Betrachtungsdimensionen hervorgebracht werden, die sich isolierten Fallbetrachtungen möglicherweise verschließen. Die komparative Analyse kann somit als „,notwendige Voraussetzung“ gesehen werden, ,um zu einer validen und methodisch kontrollierten Beschreibung und Erklärung“ (Kelle \& Kluge, 2010, S. 11) zu gelangen.

Die Interpretation von Interaktionsprozessen setzt nach Bohnsack (2010, S. 195) „Vergleichshorizonte“ voraus. Das bedeutet, dass die Interpretation eines Interaktionsausschnitts nur im Vergleich mit hypothetischen alternativen Interaktionsverläufen erfolgen kann. Da diesen alternativen Deutungen jedoch das theoretische Vorwissen, die Erfahrung und die Intuition des Interpretierenden zugrunde liegt, werden die Vergleichshorizonte des Interpretierenden durch empirisch generierte Vergleichshorizonte ersetzt, die empirisch generiert und somit intersubjektiv nachvollziehbar und überprüfbar sind (vgl. Bohnsack, 2010, S. 137). Auf diese Weise werden andere empirische Daten als Vergleichshorizonte herangezogen und dadurch das theoretische Vorwissen des Forschenden methodisch kontrolliert. Dies kann 
sowohl innerhalb eines Falls wie auch über den Fallvergleich gestaltet werden (Bohnsack, 2010, S. 137f.).

Die komparative Analyse ermöglicht die Bildung von Typen und die Kontrolle des theoretischen Vorwissens. In Hinsicht auf die Entwicklung einer Theorie können durch die Komparation zum einen jene Theoriekonstrukte ausgeschlossen werden, die nicht zu den Interpretationen der ausgewählten Realitätsausschnitte passen und zum anderen werden auf der Basis komparativer Analysen empirisch kontrollierte und theoretisch orientierte Bedingungen für das abduktive Erschließen neuer Theorieelemente erzeugt (Krummheuer \& Brandt, 2001, S. 81).

\section{Analyse von Grundvorstellungen}

In Abschnitt 2.1 wurden Grundvorstellungen auf normativer Ebene als ,didaktische Kategorie des Lehrers“ beschrieben, die ,im Hinblick auf ein didaktisches Ziel aus inhaltlichen Überlegungen hergeleitet wurde und Deutungsmöglichkeiten eines Sachzusammenhangs bzw. dessen mathematischen Kerns beschreiben“" (vom Hofe, 1995, S. 123). Dieser normativen Ebene der Sachanalyse stellt vom Hofe eine deskriptive Ebene der Analyse von Schülervorstellungen gegenüber: „Schülervorstellungen geben dabei Aufschluss über die individuellen Erklärungsmodelle des Schülers, die in das System seiner Erfahrungsbereiche eingebunden und entsprechend aktivierbar sind“" (vom Hofe, 1995, S. 123).

Vor diesem Hintergrund wurden die Leitfragen zur Analyse der normativen Verwendung von Grundvorstellungen und der deskriptiv feststellbaren Individualvorstellungen der Lernenden wie folgt formuliert:

- „Welche Grundvorstellungen sind zur Lösung des Problems aus Sicht des Lehrenden adäquat? (Normativer Aspekt)

- Welche individuellen Vorstellungen lassen sich im Lösungsversuch des Schülers erkennen? (Deskriptiver Aspekt)

- Worauf sind etwaige Divergenzen zurückzuführen, und wie lassen sich diese beheben? (Konstruktiver Aspekt)“ (vom Hofe, 1995, S. 116f.)

Leitfragen wie diese bilden die Grundlage für „,interpretative Fallanalysen“ (vom Hofe, 1992, S. 360; vgl. auch vom Hofe, 1998; vom Hofe, 1999), in denen die Bearbeitungen von Lernenden anhand von Transkripten der Lernendenkommunikation und den schriftlichen Produkten der Lernenden in Hinsicht auf ein spezifisches Forschungsinteresse analysiert werden. Die Analysen erfolgen dabei auf zwei Ebenen: Der Beschreibungsebene und der Erklärungsebene, denen die folgenden Leitfragen entsprechen: 
- „Deskriptives Nachzeichnen der subjektiven Schülerlogik. Welche subjektiven Vorstellungen bzw. Deutungsmodelle werden in den Lösungsversuchen der Schülerinnen deutlich? Inwieweit lassen sich dabei individuelle Denkmuster bzw. Lösungsstrategien nachzeichnen?

- Vergleichende Einbeziehung präskriptiver Kategorien. Inwieweit lassen sich Denkprozesse der Schülerinnen mit vorhandenen didaktischen Begriffen und Modellen erfassen und erklären?“ (vom Hofe, 1998, S. 266)

Entsprechend werden die Interaktionen der Lernenden zunächst eingehend beschrieben und in der Folge mit präskriptiven normativen Aspekten verglichen, um Divergenzen zwischen erwarteten und den deskriptiv feststellbaren Erklärungsmodellen und Vorstellungen der Lernenden aufzudecken und zu erklären. Diese Methode bietet sich an,

„wenn man sich aus deskriptiver Sicht dafür interessiert, ob Erklärungsmodelle, mit denen man Lern- bzw. Problemlöseprozesse beschreibt, tatsächlich in den Denkprozessen der Schüler die Rolle spielen, die man aus theoretischer Sicht vermutet." (vom Hofe, 1998, S. 259)

In den Arbeiten von vom Hofe $(1998 ; 1999)$ wurden mithilfe interpretativer Fallanalysen selbstorganisierte Arbeitsphasen im computergestützten Analysisunterricht untersucht, um charakteristische Schwierigkeiten bei der Entwicklung des Grenzwertbegriffs (vom Hofe, 1998) und typische Handlungsmuster in selbstgesteuerten Arbeitsphasen am Computer (vom Hofe, 1999) zu identifizieren.

In den Arbeiten von Wartha (2007) und Hafner (2012) wurde dieser methodische Ansatz angewendet, um charakteristische Fehlermuster und Fehlvorstellungen in der Bruchrechnung (Wartha, 2007) und der Prozentrechnung (Hafner, 2012) zu identifizieren und zu beschreiben.

In allen Fällen folgten die Analysen der Struktur, dass zunächst auf Grundlage von sachanalytischen Überlegungen Erwartungen an die Lösungsprozesse der Lernenden formuliert wurden (normative Ebene). Die Bearbeitungen der Lernenden wurden dann deskriptiv im Detail beschrieben und in Hinsicht auf die vermuteten individuellen Vorstellungen und Erklärungsmodelle der Lernenden interpretiert. Über einen Vergleich der normativen und deskriptiven Analysen wurden abschlieBend Folgerungen für die Unterrichtspraxis (konstruktive Ebene) abgeleitet.

\section{Beschreibung des methodischen Vorgehens in dieser Arbeit}

Das methodische Vorgehen in dieser Arbeit ist als eine Verbindung der Methoden 
der interpretativen Unterrichtsforschung und der Analyse von Grundvorstellungen zu beschreiben.

Mit dem Ziel der Identifikation, Rekonstruktion und detaillierten Beschreibung von Transferprozessen in Hinsicht auf die formulierten Forschungsfragen werden ausgewählte Bearbeitungen der Schülerinnen und Schüler auf Grundlage der in Kapitel 2 neu formulierten Leitfragen untersucht:

- Welche Transferprozesse sind zur Lösung einer neuen Anforderung erforderlich und welche Grundvorstellungen werden dafür benötigt? (Normativer Aspekt)

- Welche individuellen Transferprozesse lassen sich in den Bearbeitungen der Lernenden erkennen und welche individuellen Vorstellungen liegen diesen zugrunde? (Deskriptiver Aspekt)

- Worauf sind etwaige Divergenzen zurückzuführen und wie lassen sich diese beheben? (Konstruktiver Aspekt)

Die Auswahl der Bearbeitungen der Lernenden zur Detailanalyse folgt dem Prinzip der Komparation, indem möglichst unterschiedliche Einzelbearbeitungen miteinander in Beziehung gesetzt werden. Möglichst große Unterschiede werden dabei zum einen durch unterschiedliche Lösungen und zum anderen durch unterschiedliche Lösungswege beschrieben. Das Ziel ist es über den Vergleich kontrastierender Fälle Spezifika und Gemeinsamkeiten herauszuarbeiten, die sich einer isolierten Fallbetrachtung möglicherweise verschließen.

Dazu werden zu allen drei Erhebungszeitpunkten Bearbeitungen von Lernenden zu gleichen Aufgaben ausgewählt und analysiert. Die Analysen der Bearbeitungsprozesse werden dann in den Vergleich zu Analysen von Bearbeitungsprozessen von anderen Aufgaben desselben Erhebungszeitpunkts gesetzt. Am Ende werden die Ergebnisse der vergleichenden Analysen der drei Erhebungszeitpunkte miteinander verglichen, um den Vergleichshorizont zu erweitern und die Transferprozesse im Verlauf aller drei Erhebungszeitpunkte miteinander in Beziehung zu setzen, um Gemeinsamkeiten und Unterschiede sowie übergreifende Charakteristika herauszustellen.

Die Detailanalysen folgen dem Prinzip der Analyse von Grundvorstellungen und sind auf einer Beschreibungs- und einer Erklärungsebene organisiert: Zunächst wird auf der Beschreibungsebene durch interpretative Analysen versucht die subjektive Logik der Lernenden während der Bearbeitung nachzuzeichnen bzw. zu rekonstruieren. Diese werden dann auf der Erklärungsebene mit den zuvor auf Grundlage einer Sachanalyse beschriebenen normativ erwarteten Bearbeitungs- und Transferprozessen verglichen. 
Das methodische Vorgehen auf der Beschreibungsebene orientiert sich am Analysemuster der interpretativen Unterrichtsforschung. Es wird zunächst eine Gliederung der Bearbeitung in Szenen vorgenommen, die sich an der Bearbeitung von Teilaufgaben und einzelnen Lösungsschritten orientiert. Im Anschluss werden die Einzeläußerungen und Handlungen der Lernenden beschrieben und mit Bezug auf den Bearbeitungsprozess interpretiert. Die auf diese Weise entwickelten Interpretationen müssen sich im weiteren Verlauf der Analyse bewähren. Zum Schluss werden die tragfähigsten Interpretationen in Form von Deutungshypothesen zusammengefasst, die als Grundlage für die vergleichenden Analysen am Ende des jeweiligen thematischen Abschnitts dienen. Hierbei werden in den Interpretationen empirische Erkenntnisse zur Entwicklung des Bruchzahlbegriffs und charakteristischen Problembereichen (vgl. Abschnitt 2.2) einbezogen.

\section{Transkription der videographierten Daten}

Für die Transkription der Videodaten wird eine linearisierte Darstellung genutzt, weil diese vor dem Hintergrund des beschriebenen Forschungsinteresses zielführend ist, eine hohe Lesbarkeit verspricht und das Nachvollziehen von Unterrichtssituationen vereinfacht (vgl. Kowal \& O'Connell, 2007). Die Vorzüge von detaillierten Darstellungen, zum Beispiel der differenzierten Partiturschreibweise einer gesprächsanalytischen Arbeitstranskription, kämen in der vorliegenden Arbeit kaum zum Tragen, da das zentrale Forschungsinteresse nicht den Interaktionsstrukturen und -mustern in den Lernendenbearbeitungen gilt. Den Nachteilen der gewählten Darstellungsform, wie zum Beispiel die fehlende Möglichkeit synchrone Prozesse und Gesprächssequenzen entsprechend zu repräsentieren, wird durch die Nutzung kursiver Kommentare in den linearen Transkripten begegnet (vgl. Dresing \& Pehl, 2020; Salle, 2015). Auf diese Weise können nicht strukturell im Transkript repräsentierbare Informationen angemessen dargestellt werden. Die Zeilennummern eines Transkripts sind fortlaufend vergeben und beginnen stets bei 1 . Auslassungen werden im Transkript oder im Text kenntlich gemacht.

Für die Transkripte werden folgende Zeichen und Ausdrücke genutzt:

normaler Text stellt gesprochenen Text dar

(kursiver Text in Klammern) stellt Kommentare zum Transkript dar . . symbolisiert eine kurze Pause von maximal 2 Sekunden

. . . symbolisiert eine Pause von maximal 5 Sekunden (längere Pausen werden als Kommentare angegeben) 
Open Access Dieses Kapitel wird unter der Creative Commons Namensnennung 4.0 International Lizenz (http://creativecommons.org/licenses/by/4.0/deed.de) veröffentlicht, welche die Nutzung, Vervielfältigung, Bearbeitung, Verbreitung und Wiedergabe in jeglichem Medium und Format erlaubt, sofern Sie den/die ursprünglichen Autor(en) und die Quelle ordnungsgemäß nennen, einen Link zur Creative Commons Lizenz beifügen und angeben, ob Änderungen vorgenommen wurden.

Die in diesem Kapitel enthaltenen Bilder und sonstiges Drittmaterial unterliegen ebenfalls der genannten Creative Commons Lizenz, sofern sich aus der Abbildungslegende nichts anderes ergibt. Sofern das betreffende Material nicht unter der genannten Creative Commons Lizenz steht und die betreffende Handlung nicht nach gesetzlichen Vorschriften erlaubt ist, ist für die oben aufgeführten Weiterverwendungen des Materials die Einwilligung des jeweiligen Rechteinhabers einzuholen.

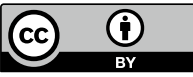

